\title{
Intraoperative use of point of care ultrasonography (POCUS) for stat cesarean delivery with refractory hypoxemia
}

Nicholas J. Schott ${ }^{1 *}$, Dana Leonelli ${ }^{2}$ and Susan McElroy ${ }^{1}$

*Correspondence: schottnj@upmc.edu

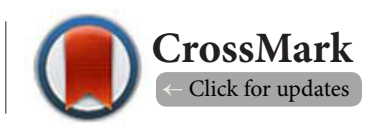

'Department of Anesthesiology, University of Pittsburgh, USA.

${ }^{2} \mathrm{~S}$ chool of Nursing, University of Pittsburgh, USA.

\begin{abstract}
A 30-year-old female at 24 weeks gestation presented with headache, hypertension and blurry vision. She was diagnosed with pre-eclampsia on chronic hypertension. An obstetrical emergency was called due to fetal heart rate decreasing to a nadir of 40bpm and the patient was taken for an emergent cesarean delivery. Prior to induction of anesthesia, the $\mathrm{SpO}_{2}$ was $92 \%$. A rapid sequence induction was conducted with confirmed intubation however, $\mathrm{SpO}_{2}$ decreased to $71 \%$ over time. The patient was ventilated manually and was given bronchodialators. However, oxygenation did not improve. A portable point-ofcare ultrasound (POCUS) device was brought into the operating room. Windows obtained suggested that the patient had gross pulmonary edema resulting from begining cardiac failure. Given the findings, 1:1 crystalloid replacement was undertaken rather than the traditional 3:1 replacement for crystalloid to blood loss ratio. Blood pressure was reduced with medications including volatile agents. The patient's $\mathrm{SpO}_{2}$ slowly improved and by the end of the surgical procedure. Re-imaging of the pleural views revealed improvement of interstitial pulmonary fluid. The patient remained intubated post-operatively. The patient was extubated on POD 2 and eventually discharged home. Peri-operative POCUS was used to help aid in a diagnosis of an emergent cesarean delivery with ongoing hypoxia. Multiple disciplines including obstetrics, anesthesia and critical care were able to visualize her problems and collaborate with plan for management and post operative care.
\end{abstract}

Keywords: Cesarean, hypoxemia, eclampsia, ultrasound

\section{Introduction}

Severe pre-eclampsia is universally known in obstetrical anesthesia and can lead to multiple complications for mother and child. Pertinent concerns to the anesthesia team are effects on maternal pulmonary, cardiac, neurologic and coagulation systems. Concurrently, treatments initiated for severe pre-eclampsia, such as magnesium sulfate, can disrupt physiology with specific regard to cardiac, pulmonary, and neuromuscular systems. Some features of severe pre-eclampsia may be life-threatening. Timely evaluation may be critical for a patient when delivery of a fetus is emergent and a cesarean delivery is required before any anesthesia assessment has been made. We present a case of a parturient for emergent cesarean delivery with resulting hypoxemia in which was diagnosed and managed by the use of intraoperative point of care ultrasound.

\section{Case presentation}

A 30-year-old, $131 \mathrm{~kg}$ (BMI 43), G6P5 female at 24 weeks gestation was transferred from an outlying facility with chief complaint of headache, hypertension and blurry vision. She was diagnosed with superimposed pre-eclampsia (SIPE) on chronic hypertension. Treatment included admission into the intensive care unit (ICU) for intravenous pharmacologic blood pressure control. The blood pressure on admission was $220 / 140 \mathrm{mmHg}$. An obstetrical emergency was called immediately upon admission due to fetal heart rate decreasing to nadir of $40 \mathrm{bpm}$ when examined in the ICU and the patient was taken for an emergent cesarean delivery. 


\section{Intraoperative course}

Prior to induction of anesthesia, it was verified that the patient had no known allergies, a NPO status of 9.5 hours and after visualization, the airway was classified as a Mallampati 3. The patient had one 18 gauge peripheral IV with $2 \mathrm{gm}$ Magnesium sulfate infusing which was stopped prior to induction. Initial $\mathrm{SpO}_{2}$ was $92 \%$ on $6 \mathrm{~L} \mathrm{O}_{2}$ facemask. The patient was placed on the operating room table and pre-oxygenated with $100 \%$ free flow oxygen for approximately one minute. At induction of general anesthesia, her oxygen saturation was $99 \%$, blood pressure $204 / 129 \mathrm{mmHg}$ (after IV hydralazine administration by the Obstetrics team), and a pulse of 95 beats per minute. A rapid sequence induction with cricoid pressure was conducted utilizing propofol $200 \mathrm{mg}$, rocuronium $5 \mathrm{mg}$ and succinylcholine $160 \mathrm{mg}$. Initial intubation was unsuccessful using a MAC 3 blade with inability to visualize the glottis opening as the velecula was distal to the laryngoscope blade. Successful, atraumatic intubation (Cormack Grade 3) was achieved using a video laryngoscope blade with end tidal $\mathrm{CO}_{2}$ confirmation and bilateral breath sounds. Sevoflurane was started at $1.5 \%$ and $50 \% \mathrm{O}_{2}$ with $50 \%$ $\mathrm{N}_{2} \mathrm{O}$ gas flow. Blood pressure decreased to $175 / 92 \mathrm{mmHg}$ with a heart rate if 90 beats per minute.

Immediately after the start of the procedure, the patient's $\mathrm{SpO}_{2}$ dropped to $71 \%$ and the patient was started on $100 \%$ oxygen. The patient was ventilated manually with peep $7-10 \mathrm{cmH}_{2} \mathrm{O}$ and was given 8 puffs of Albuterol via the endotracheal tube. The fetus was delivered 1.5 minutes after induction of anesthesia. The maternal blood pressure decreased to $125-150 \mathrm{mmHg}$ systolic over $70-100 \mathrm{mmHg}$ diastolic pressures post delivery. In an attempt to increase the effectiveness of manual ventilation, the patient was given 40mg Rocuronium; however, ventilation and oxygenation did not improved. A right radial arterial line was placed to monitor hemodynamic changes and obtain arterial blood gases for analysis. The first arterial blood gas demonstrated a mixed respiratory and metabolic acidosis with $\mathrm{pH} 7.19, \mathrm{pCO}_{2}$ 55.1, $\mathrm{pO}_{2} 70.1, \mathrm{HCO}_{3}$ 20.3, Base Deficit -6, $\mathrm{SO}_{2} 87.9 \%$, Lactate 1.2, and Hemoglobin 11.6.

The oxygen saturation remained between $71-86 \%$ after administration of bronchodilator therapy, high levels of positive end expiratory pressure, and $100 \%$ oxygen using manual ventilation. Differential diagnosis initially included bronchospasm, pneumothorax, pulmonary edema, hemothorax, atelectasis and pneumonia. In order to assist in diagnosing and planning further anesthetic treatment, the point-of-care ultrasound was brought into the operating room. The FATE protocol was used (Figure 1). Windows obtained suggested that the patient had a small pericardial effusion, left ventricular hypertrophy and gross pulmonary edema as illustrated by multiple "b-lines" in pleural view (Images 1-3). Given the findings on the pointof-care ultrasound and concern for evolving congested heart failure, a 1:1 crystalloid replacement was undertaken rather than the traditional 3:1 replacement. Discussion of diuretic use was made, however given the changing hemodynamics use was withheld. Mild secretions were obtained through endotracheal

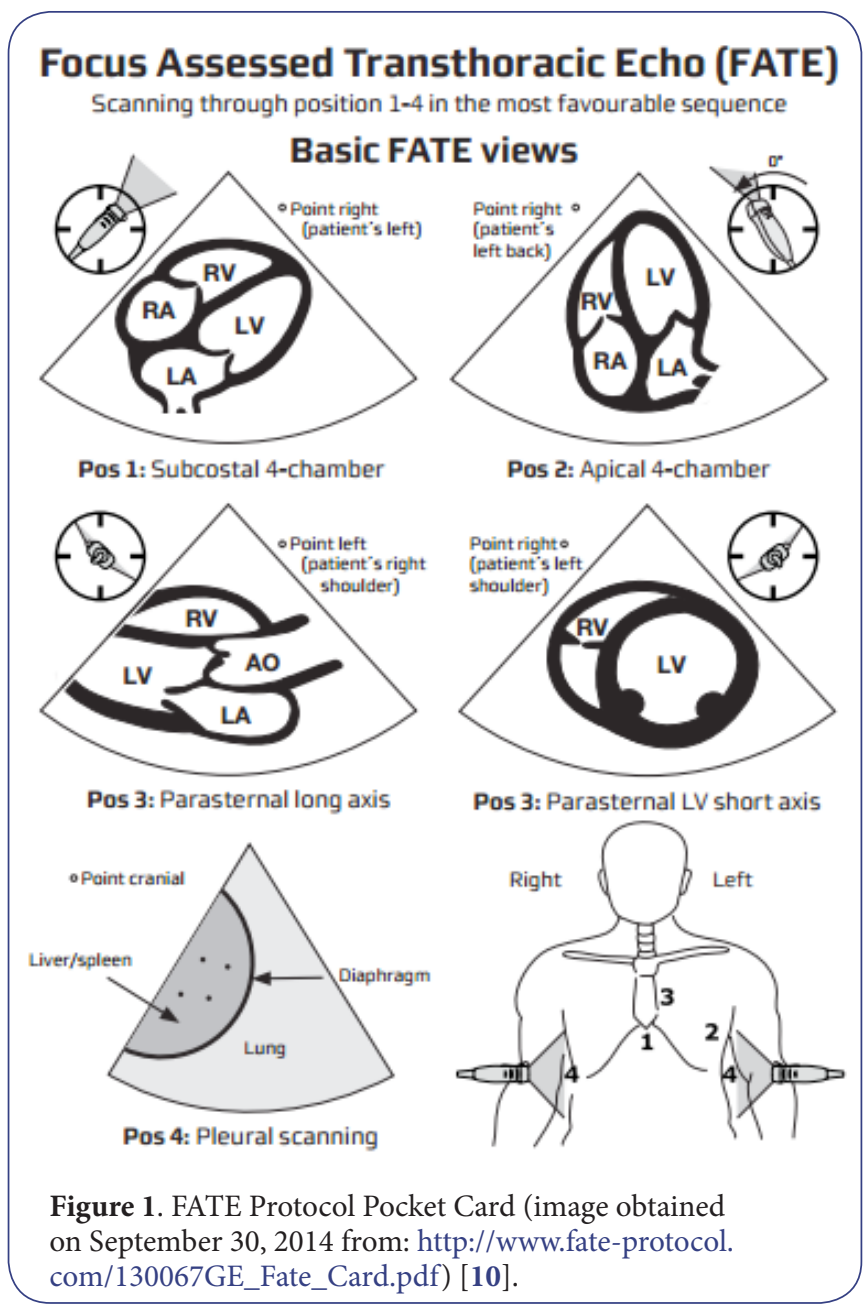

suctioning. The patient's $\mathrm{SpO}_{2}$ slowly improved and by the end of the surgical procedure, the patient's $\mathrm{SpO}_{2}$ was between $88-91 \%$.

The ICU team was notified of the patient's condition and diagnosis. The patient's oxygen saturation, arterial blood pressure and EKG was monitored and remained stable without treatment during transfer to the ICU. A chest radiograph obtained 2 hours postoperatively confirmed pulmonary edema.

\section{Results and postoperative course}

The patient remained intubated and sedated on assist control ventilation in the ICU for 48 hours postoperatively. A formal transthoracic echocardiogram obtained on postoperative day 1 further supported the diagnosis; showing left ventricular hypertrophy, a small pericardial effusion and signs of vascular congestion. On postoperative day 2 , after noting improvement in chest radiograph, the sedation was discontinued and the patient was extubated. The patient's blood pressure and pulmonary status improved within days with appropriate management. The patient continued medical treatment as an inpatient until postoperative day 5 and was discharged home. The neonate was alive and supported in the neonatal intensive care unit. 


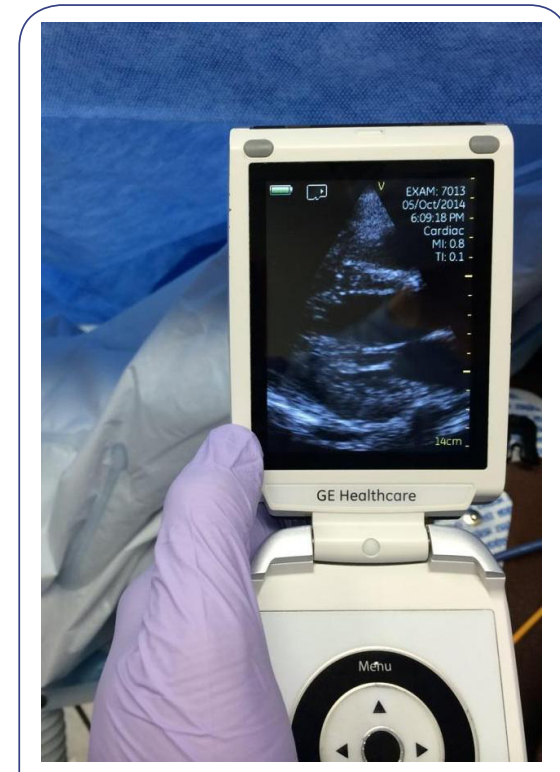

Image 1. Portable POCUS using GE Vscan undergoing FATE protocol on intraoperative machine use. Images show $\mathrm{LVH}$ and a small pericardial effusion.

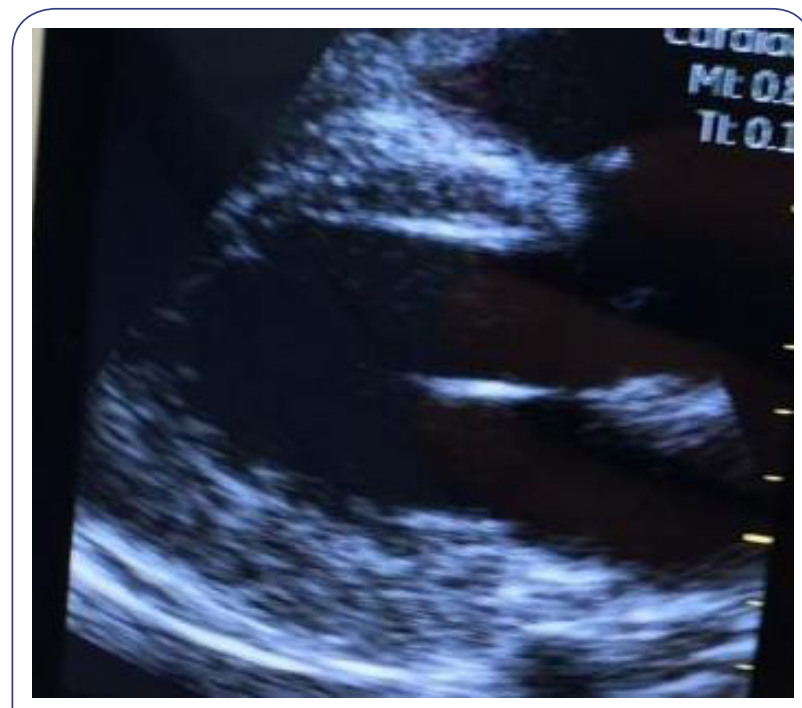

Image 2. Portable POCUS using GE Vscan. Image shows enlarged view of Image 1 demonstrating $\mathrm{LVH}$ and a small pericardial effusion.

\section{Discussion}

Ultrasound has become an indispensable tool in the anesthesiologist's armamentarium. A surface echogenic machine is vital for safe placement of regional anesthesia and vascular accesses. However, portable transthoracic echocardiography (TTE) has not been fully appreciated for its perioperative use, despite widespread popularity in the fields of emergency and critical care medicine. Advances in ultrasound technology have solved

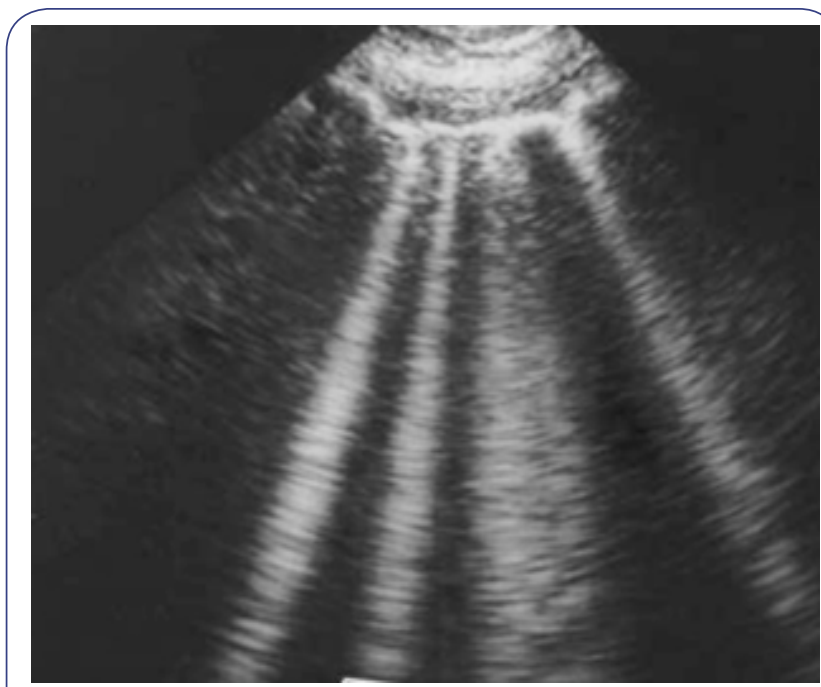

Image 3. This window is of the right pleural space at T7/ T8. Rib shadows seen at lateral margins. Multiple "B-lines", shown by white vertical lines in lung intercostal field, indicating intestinal fluid with clinical correlate of pulmonary edema in the patient.

portability and accessibility issues; portable ultrasound devices are now available in many fields of clinical practice $[\mathbf{1 , 2}]$. Previous studies have validated the use of portable TTE by multiple providers in the intensive care unit and other settings [3-5].

No formal guidelines for anesthesiologists' use of portable TTE to perioperatively assess patients have been established. However, the technology is quickly being adopted for training and use in acute care specialties $[\mathbf{1 , 2 , 4}]$. TTE serves as another physical examination tool with which to learn about a patient's physical condition, especially in acute settings such as emergency cases. Where physiology can change dramatically at any moment, as in a parturient at time of delivery, a readily-accessible and non-invasive tool such as portable TTE can play a substantial role in patient assessment and management. Incorporation of our program's TTE curriculum into anesthesiology in multiple facets has given another option to provide anesthesiology providers with the necessary knowledge and skills to manage patients with cardiopulmonary instability.

In obstetrical anesthesia, the use of POCUS may be even more valuable for patient assessment, diagnosis, monitoring and treatment. The majority of obstetrical patients have few comorbidities and are unlikely to have a previous echocardiographic study. Not many studies have looked at term and near-term parturients in regard to cardiac function. When a patient presents with a possible cardiopulmonary disease or complication, the use of POCUS could be critical. This is especially true when in an emergent obstetrical situation.

Other helpful information obtained by the use of bedside ultrasonography is specific and targeted information that may be helpful for intraoperative diagnostics. This includes assessment of valvular disease (stenosis, regurgitation), pressure 
estimation (pulmonary, transvalvular, intracardiac) as well of physical properties of the heart (segmentary wall motion, ejection fraction and caval dimensions). This may aid in an obstetric patient such as applied in this case where increased blood pressure may have resulted in increased pulmonary arterial and pulmonary wedge pressures in the hypertrophied heart. This could lead to potential concerns of hypotension by effectively reduced preload. Effects are then potentially compounded by medications such as induction agents or Magnesium given for pre-eclampsia albeit not seen in this case.

Fluid administration was discussed with the obstetric team. Haste use of diuretics was contemplated. However, acute blood loss during the procedure risked potential to worsen hemodynamic instability from the diuresis. Therefore, administration of 1:1 crystalloid to estimated blood loss was instituted. This approximation was intended to maintain arterial volume without worsening acute pulmonary interstitial fluid overload. Once the procedure was close to completion and blood loss was minimized, further crystalloid administration was halted until a follow-up POCUS was conducted. The improvement helped confirm the diagnosis and lead to optimize management of the patient.

Overall in the case presented, quick evaluation of a patient undergoing a general anesthetic cesarean delivery was determined using POCUS assistance. Given the patient's ongoing pre-eclampsia, focus was directed to the cardiopulmonary system. The intraoperative POCUS exam helped make a clinical decision that the patient was experiencing cardiopulmonary edema worsened by her acute elevated hypertension prior to delivery.

\section{Conclusions}

In respect to this case presentation, we conclude that the use of POCUS is helpful in the hands of a trained provider. Additionally, use of POCUS can provide a concrete visual to communicate with multiple acute care teams for a critically ill patient. Longitudinal and multiple provider assessment of this modality requires further study.

\section{Competing interests}

The authors declare that they have no competing interests.

\section{Authors' contributions}

Nicholas J. Schott, MD contributing to writing, editing and approved of manuscript. Dana Leonelli, BSN contributed writing, editing and approved of final manuscript. Susan McElroy, DO contributed writing, editing and approved of final manuscript.

\section{Publication history}

Editor: Pietro Di Pasquale, University of Palermo, Italy.

Received: 11 March 2016 Revised: 25 April 2016

Accepted: 12 May 2016 Published: 20 May 2016

\section{References}

1. Jensen MB, Sloth E, Larsen KM and Schmidt MB. Transthoracic echocardiography for cardiopulmonary monitoring in intensive care. Eur J Anaesthesiol. 2004; 21:700-7. I PubMed

2. Melniker LA, Leibner E, McKenney MG, Lopez P, Briggs WM and Mancuso CA. Randomized controlled clinical trial of point-of-care, limited ultrasonography for trauma in the emergency department: the first sonography outcomes assessment program trial. Ann Emerg Med. 2006; 48:227-35. | Article | PubMed

3. Vignon P, Dugard A, Abraham J, Belcour D, Gondran G, Pepino F, Marin B, Francois $B$ and Gastinne $\mathrm{H}$. Focused training for goal-oriented handheld echocardiography performed by noncardiologist residents in the intensive care unit. Intensive Care Med. 2007; 33:1795-9. | Article | PubMed

4. Mahler SA, Swoboda TK, Wang H and Arnold TC. Dedicated emergency department ultrasound rotation improves residents' ultrasound knowledge and interpretation skills. J Emerg Med. 2012; 43:129-33. | Article I PubMed

5. Canty DJ, Royse CF, Kilpatrick D, Bowman L and Royse AG. The impact of focused transthoracic echocardiography in the pre-operative clinic. Anaesthesia. 2012; 67:618-25. I Article | PubMed

6. Neelankavil J, Howard-Quijano K, Hsieh TC, Ramsingh D, Scovotti JC, Chua $\mathrm{JH}, \mathrm{Ho} \mathrm{JK}$ and Mahajan A. Transthoracic echocardiography simulation is an efficient method to train anesthesiologists in basic transthoracic echocardiography skills. Anesth Analg. 2012; 115:1042-51. I Article | PubMed

7. Kimura BJ, Amundson SA, Phan JN, Agan DL and Shaw DJ. Observations during development of an internal medicine residency training program in cardiovascular limited ultrasound examination. J Hosp Med. 2012; 7:537-42. | Article | PubMed

8. Barsuk JH, Cohen ER, Vozenilek JA, O'Connor LM, McGaghie WC and Wayne DB. Simulation-based education with mastery learning improves paracentesis skills. J Grad Med Educ. 2012; 4:23-7. I Article I PubMed Abstract I PubMed FullText

9. Baranauskas MB, Margarido CB, Panossian C, Silva ED, Campanella MA and Kimachi PP. Simulation of ultrasound-guided peripheral nerve block: learning curve of CET-SMA/HSL Anesthesiology residents. ReV Bras Anestesiol. 2008; 58:106-11. I Article I PubMed

10. Jammal M, Milano P, Cardenas R, Mailhot T, Mandavia D and Perera P. The diagnosis of right heart thrombus by focused cardiac ultrasound in a critically ill patient in compensated shock. Crit Ultrasound J. 2015; 7:6. | Article | PubMed Abstract | PubMed FullText

11. Atkinson PR, McAuley DJ, Kendall RJ, Abeyakoon O, Reid CG, Connolly $\mathrm{J}$ and Lewis D. Abdominal and Cardiac Evaluation with Sonography in Shock (ACES): an approach by emergency physicians for the use of ultrasound in patients with undifferentiated hypotension. Emerg Med J. 2009; 26:87-91. | Article | PubMed

\section{Citation:}

Schott N.J, Leonelli D and McElroy S. Intraoperative use of point of care ultrasonography (POCUS) for stat cesarean delivery with refractory hypoxemia.

J Anesthesiol Clin Sci. 2016; 5:5.

http://dx.doi.org/10.7243/2049-9752-5-5 